\title{
The aerodynamic effects on a cornering Ahmed body
}

\author{
James Keogh ${ }^{\mathrm{a}}$, Tracie Barber ${ }^{\mathrm{a}}$, Sammy Diasinos ${ }^{\mathrm{b}}$, Graham Doig ${ }^{\mathrm{c}}$ \\ a School of Mechanical and Manufacturing Engineering, UNSW, Sydney, NSW 2052, Australia \\ ${ }^{\mathrm{b}}$ Department of Engineering, Macquarie University, Australia

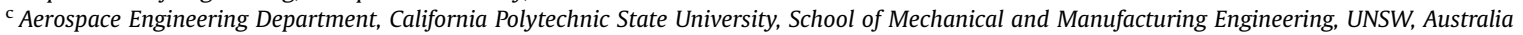

Keywords:

Aerodynamics

Automotive

Cornering

Computational fluid dynamics

Bluff body

\begin{abstract}
A B S T R A C T
As a vehicle travels through a corner, the flowfield observed from the vehicle's frame of reference becomes curved. This condition results in the relative flow angle and freestream velocity changing both across the width and along the length of the body. Wall-resolved Large Eddy Simulations were used to simulate a simple vehicle shape through three different radii corners. The variable flow angle and acceleration affected the pressure distribution along either side of the body and caused an increase in the size of the outboard C-pillar vortex, and an inboard decrease. Furthermore, an outboard extension of the separation bubble at the bluff trailing face resulted in a gentler downwash angle off the backlight surface, with the opposite occurring inboard. At a Reynolds number of $1.7 \times 10^{6}$, a $19.2 \%$ increase in aerodynamic drag occurred for a five car-length radius corner when compared to the straight-line condition. In addition, a yawing moment acted against the rotation of the body through the corner, and a side force acted towards the centre of the corner. An exponential trend related the curvature of a vehicle's path to the increase in aerodynamic drag, with a linearity exhibited for the increase in yawing moment and side force.
\end{abstract}

\section{Introduction}

Technical development in the automotive industry continues to increase the performance capabilities of vehicles. As dynamic capabilities improve, tighter manoeuvres can be achieved at higher speeds, and will result in the aerodynamic effects having a greater contribution towards overall performance. Some vehicles use these higher speeds beneficially to create large amounts of aerodynamic downforce and further enhance cornering speeds (Toet, 2013; Katz, 2006). Despite this, designs will typically only be analysed in the straight-line condition and yaw (Toet, 2013). Recent studies in the field have considered a wider variety of conditions to assess real-world aerodynamic performance and this has resulted in investigations into conditions such as transient cross-winds and travelling through a corner (Tsubokura et al., 2012; Okada et al., Fujita; Nara et al., 2014; D'Hooge et al., 2014; Keogh et al., 2015a). Corners, in particular, have proved problematic due to the difficulty in recreating the conditions

\footnotetext{
Abbreviations: $C_{\mathrm{M} \psi}$, Yaw moment coefficient; $C_{S}$, side force coefficient; COR, centre of rotation; $L$, body length, $1044 \mathrm{~mm}$; $P$, static pressure; $Q, Q$-criterion normalised according to $U_{\infty} / L ; R$, corner radius; $U_{\infty}$, freestream velocity; $u, x$-component of velocity; $v, y$-component of velocity; $w, z$-component of velocity; $\kappa$, curvature $\left(L^{-1}\right) ; \Psi$, Yaw angle $\left(^{\circ}\right) ; \omega$, angular velocity ( $\left.\mathrm{rad} / \mathrm{s}\right) ; \Omega_{x}, X$-vorticity normalised according to $U_{\infty} / L$

E-mail address: j_keogh@live.com.au (J. Keogh).
}

experimentally (Toet, 2013). There have been previous investigations into the cornering condition, but none have detailed the range of specific flow mechanisms responsible for the change in aerodynamic forces observed.

Fig. 1 shows the freestream flow characteristics for a vehicle in the steady-state cornering condition. The car itself is considered to have a constant angular velocity about a fixed external point which is representative of a constant radius, steady-state corner. The velocity of the flow relative to the car, and the relative dynamic pressure of the flow, will increase with distance from this external point. In addition the relative angle (effectively yaw) of the flow will vary along the length of the car. In the specific condition shown it can be observed that the front and rear have opposite angles of yaw. How a vehicle travels through a corner will vary for different vehicles and driving styles. The condition investigated in the present work positions the body tangent to the curvature of its path at its centre, as can be observed in Fig. 1.

There have been previous experimental attempts to replicate the cornering condition in a wind tunnel with the use of bent models (Gordes, 2005), and curved test sections (Bird et al., 1951), but these methods are not capable of representing all aspects of true cornering flow. More recently there have been developments toward a unique new type of wind tunnel which is designed to be capable of accurately recreating the cornering condition (Keogh et al., 2016) however this project remains in its infancy, and leaves 


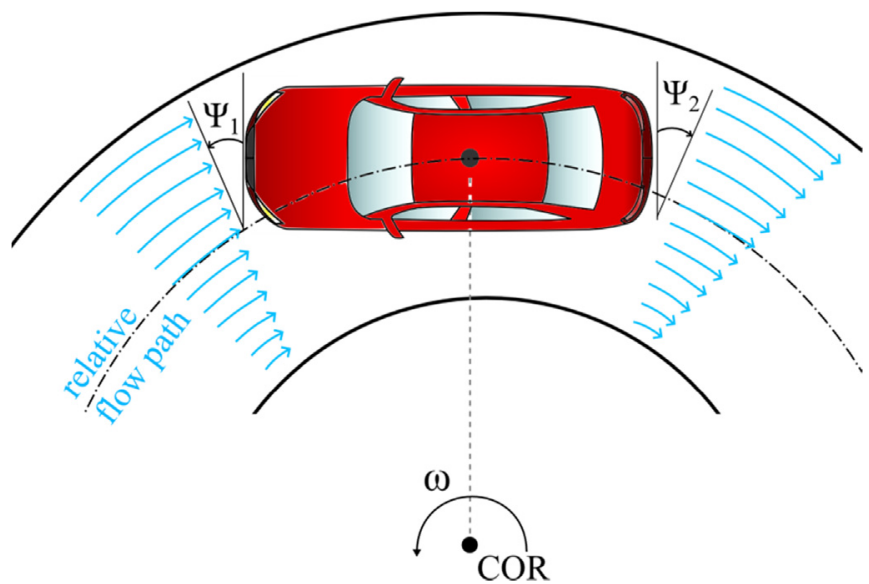

Fig. 1. The steady-state cornering flowfield as observed by a vehicle.

aerodynamicists to rely on numerical simulation (Keogh et al., 2015b).

Previous published investigations into the cornering condition have demonstrated the importance of evaluating the high speed cornering condition during the aerodynamic design phase for a passenger vehicle (Tsubokura et al., 2012; Okada et al., Fujita). These studies considered two medium-sized sedan geometries and focussed on the yawing moment which damped the rotation of the vehicle through its curved path, as well as the side force. Large Eddy Simulation was used for the numerical component of this investigation, with a Smagorinsky Subgrid-Scale model. The most influential geometric variation between the two vehicle geometries was the amount of open space around the wheel in the wheel well. This simple geometric difference caused a $49 \%$ variation in Yawing moment - with the increased space around the wheels increasing pressure losses, which enhanced the moment. The investigation related all findings to an instantaneous yaw angle at the vehicle centre, and neglected to address the freestream curvature effects caused by this type of motion.

Another prior numerical study considered an isolated inverted wing travelling in the path of a corner (Keogh et al., 2015a). The work identified significant changes to the structure of the vortical wake forming downstream and attributed these to the yaw angle of the flow across the endplates. A side force, yawing moment, and rolling moment also occurred due to a local Reynolds number increase across the span and the angle of the flow. The potential cumulative effects this could have for an entire car were highlighted through an analysis of the downstream vortex paths in the wake.

In the public domain research in automotive aerodynamics has typically favoured simple bodies over detailed car geometries. This ensures that conclusions are not likely to be geometry specific and that discoveries will likely be common aerodynamic characteristics that are widely applicable (Le Good and Garry, 2004). The Ahmed body is one of the most commonly studied simple bodies, first analysed experimentally in 1984 (Ahmed et al., 1984). The geometry was designed to maintain attached flow over the front of the vehicle and permit detailed investigation into flow features occurring over the sloping rear face, which is a common feature on hatchbacks/fastbacks. As this is the geometry selected for the present study it is appropriate to introduce the main flow features.

The wake is highly unsteady due to the blunt trailing face, but the time-averaged structure has been investigated in detail and is closely related to the pressure component of the drag (Ahmed et al., 1984; Strachan et al., 2007; Lienhart and Becker, 2003; Krajnović and Davidson, 2004, 2005a, 2005b; Minguez et al., 2008; Wang et al., 2013; Guilmineau et al., 2011; Serre et al., 2013; Bayraktar et al., 2001;

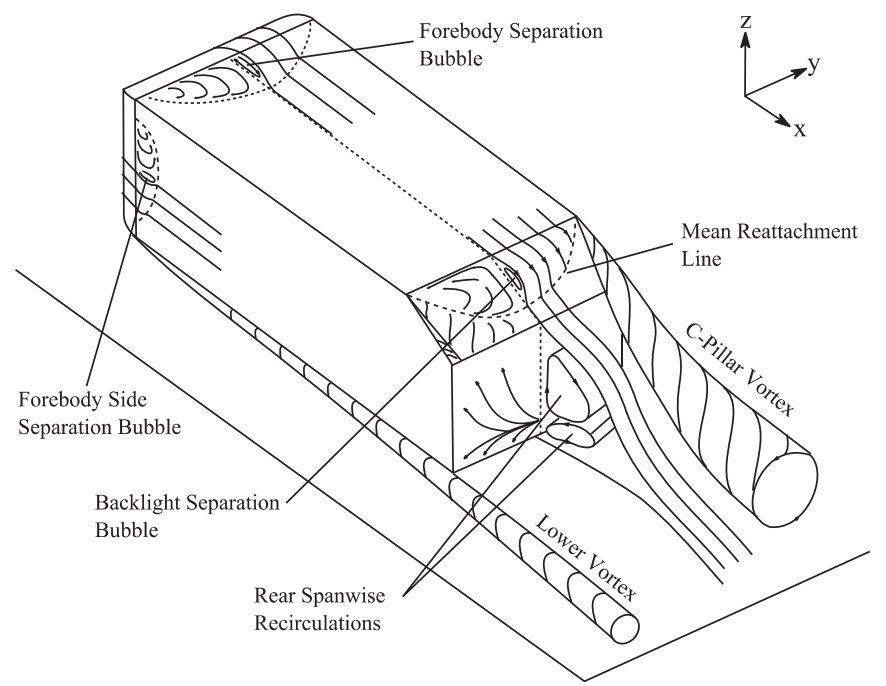

Fig. 2. An adaption of figure from Ahmed et al. (1984) detailing the prominent flow structures for the $25^{\circ}$ backlight angle.

Conan et al., 2011; Thacker et al., 2012; Joseph et al., 2012). This structure consists of both longitudinal and spanwise structures indicated in Fig. 2.

The flow down the centre of the rear sloping angle, referred to as the backlight, detaches at the start of the surface and reattaches prior to the trailing edge, forming a separation bubble, as shown in Fig. 2. Due to the lower pressure over the backlight, the shear layer from the side of the body forms a longitudinal vortex at the Cpillar location. From a backlight angle of $12.5-30^{\circ}$, the size of this vortex increases and aids in promoting the reattachment of the separation bubble. At $35^{\circ}$ the separation bubble fails to reattach and the vortex bursts (Ahmed et al., 1984; Strachan et al., 2007; Lienhart and Becker, 2003). The most commonly investigated backlight angle is $25^{\circ}$ and this is due, in part, to the difficulty associated with accurately capturing the flowfield - particularly the separation and reattachment of the flow over the rear angle. The popularity of this angular configuration made it favourable for the present study and enabled any changes that were recognised due to cornering could be compared to the findings of a wide range of previous studies (Ahmed et al., 1984; Strachan et al., 2007; Lienhart and Becker, 2003; Krajnović and Davidson, 2004, 2005a, 2005b, 2005c; Minguez et al., 2008; Wang et al., 2013; Guilmineau et al., 2011; Serre et al., 2013; Bayraktar et al., 2001; Conan et al., 2011; Thacker et al., 2012; Joseph et al., 2012).

Subsequent experimental studies have adopted similar methodologies to that of the initial study by Ahmed et al. (1984). (Lienhart and Becker, 2003) conducted an experimental study of the original configuration with the benefit of a more modern facility and found close correlation with the initial study.

Through Wall-Resolved Large Eddy Simulation (LES) (Krajnović and Davidson 2004, 2005a, 2005b) were the first to identify the existence of lower vortices, and present an accurate threedimensional understanding of the flow structure. These lower vortices occur along either side of the lower surface due to an increase in pressure from boundary layer growth along the underside of the body. Experimental studies predominantly place support struts underneath the model which inhibit the development of these structures, and hence their identification. These were initially dismissed as being of little aerodynamic significance, but have received more attention in recent publications (Strachan et al., 2007; Wang et al., 2013).

Various LES and DES models have been used to simulate the unsteady flow structure around the Ahmed body. Particularly LES has proved to be superior in capturing both longitudinal and 
spanwise structures (Krajnović and Davidson, 2004, 2005a, 2005b; Minguez et al., 2008). The use of DES over the more computationally demanding LES has aided in achieving a solution in a much shorter time-frame, but it has failed to offer the same degree of accuracy - particularly in capturing the separation and reattachment of the flow down the backlight surface (Guilmineau et al., 2011; Serre et al., 2013).

The present work focusses specifically on the aerodynamic changes that occur during cornering and how these differ from the more-familiar straight-line condition. Through this analysis an investigation into the underlying physical causes of time-averaged cornering-specific flow phenomena was conducted.

\section{Numerical method}

Wall-resolved Large Eddy Simulations (LES) were validated against the experimental results of Lienhart and Becker (2003). This was a comprehensive experimental survey of the flow structures developing around the body which was compiled into a reference database. Their experiments were conducted in a 3/4 open test-section at a length-based Reynolds number of $2.78 \times 10^{6}$, with an inlet velocity of $40 \mathrm{~m} / \mathrm{s}$. The wind tunnel used in their experiments had a low turbulence intensity $(0.25 \%)$ which permitted the use of a uniform inlet velocity profile in the numerical model. The extents of the computational domain for the validation case matched the wind tunnel nozzle cross-sectional dimensions, with zero-shear slip walls placed on the roof and side walls of the domain, and a stationary ground plane employed. Due to the blockage ratio of $4.3 \%$ in the validation simulation, a constraining effect resulted in an increase in the magnitude of aerodynamic forces - which is discussed in further detail later in this section. In the experiments the boundary layer was tripped using a grit strip located on the nose, which allowed the assumption of a fully turbulent boundary layer. The cylindrical struts used to hold the wind tunnel model in the experiments were also included in the validation computational model.

In all other cases subsequent to the validation simulation the model was placed in a 'floating' configuration and the domain size was increased to give a blockage ratio of $1.0 \%$ - further detail is provided in Section 3. Translational and rotational motion (where appropriate) of the ground plane was also included in these cases such that the motion of the ground relative to the freestream flow was zero (Krajnović and Davidson, 2005c; Barber et al., 2002). The velocity of the flow varied throughout the domain in the cornering condition due to the non-inertial reference frame, the tangential velocity at the exact centre of the body remained constant for all conditions at $25 \mathrm{~m} / \mathrm{s}$.

For LES the guidelines proposed by Pope (2000) recommend that $80 \%$ of the turbulent energy anywhere in the domain must be resolved for an accurate simulation. For a similar geometry (Krajnović and Davidson, 2004) calculated an estimate of the necessary spatial resolution for a real-world vehicle's Reynolds number of $18 \times 10^{6}$, and discovered that $6.1 \times 10^{8}$ cells would be required - a number that remains far from practical in most instances. As a result, this study employed the same methodology adopted in previous numerical studies, using a reduced Reynolds number. This prevented a prohibitive computational expense and, as this geometry exhibits moderately-low sensitivity to Reynolds number $(R e)$ in this range (Joseph et al., 2012), was also been deemed appropriate in previous studies. The use of a $R e$ of $1.7 \times 10^{6}$ permitted the use of a more manageable final grid size of $30 \times 10^{6}$ cells while maintaining a low blockage ratio - grid details are shown in Fig. 3. The maximum $y^{+}$value remained below 1 over the ground and body surfaces, and an average $\Delta x^{+}=95.3$, $\Delta y^{+}=31.3$ and $\Delta z^{+}=30.1$ were maintained. The recommendations of Piomelli and Chasnov (1996) are a first cell height at the wall giving a $y^{+}<1$, and a streamwise and spanwise resolution of $\Delta x^{+}=50-150$ and $\Delta z^{+}=15-40$. As such, the grid was designed to ensure these guidelines were adhered to in the critical regions of the backlight surface and near the edges of the body. Coarse initial simulations were used to determine the final meshing strategy and allowed informed decisions on grid resolution.

The commercial solver ANSYS Fluent 14.5 was used for all simulations. The SGS stress tensor model selected was the Smagorinsky-Lily model (Smagorinsky, 1963) where a mixing length parameter is used to approximate turbulent length scales. A fixed Smagorinsky constant $\left(C_{s}\right)$ of 0.1 was adopted, which has demonstrated good correlation with experimental results in prior studies (Krajnović and Davidson, 2004, 2005a, 2005b). The SIMPLEC pressure-velocity coupling scheme was selected for efficiency, along with a bounded central differencing scheme for momentum, second-order accuracy for pressure, and a bounded second order implicit transient formulation.

Time was non-dimensionalised according to the time taken for a single fluid particle to travel the length of the body. A time-step of $1 \times 10^{-4} \frac{L}{U_{\infty}} \mathrm{s}$ ensured a maximum CFL number of 0.95 for all simulations. Prior studies have demonstrated the complex flowfield of this particular body, with different flow features exhibiting transient behaviour operating across a range of timescales

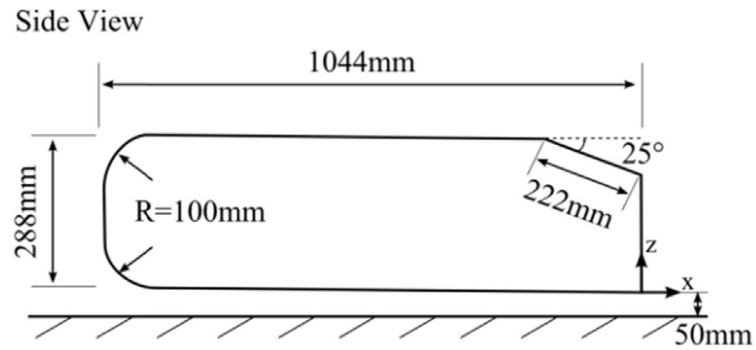

Bottom View
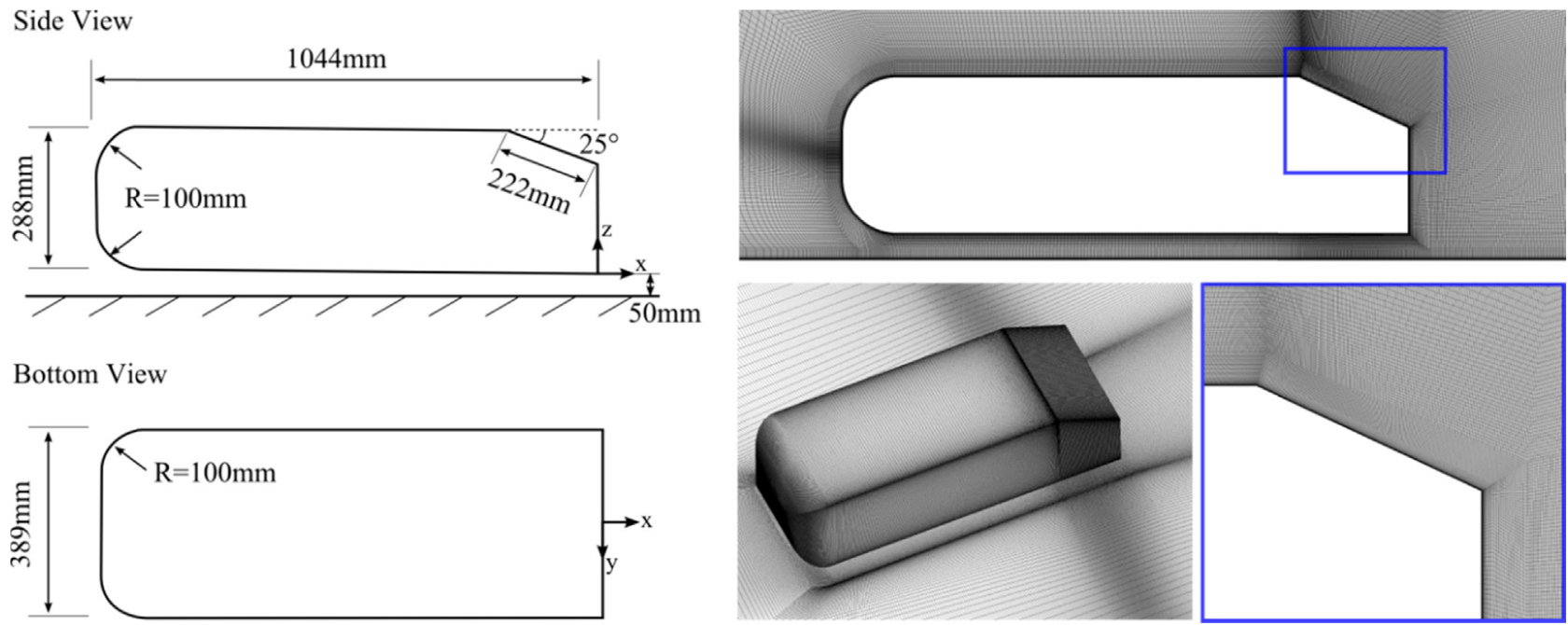

Fig. 3. Overview of the geometry and meshing strategy employed. 

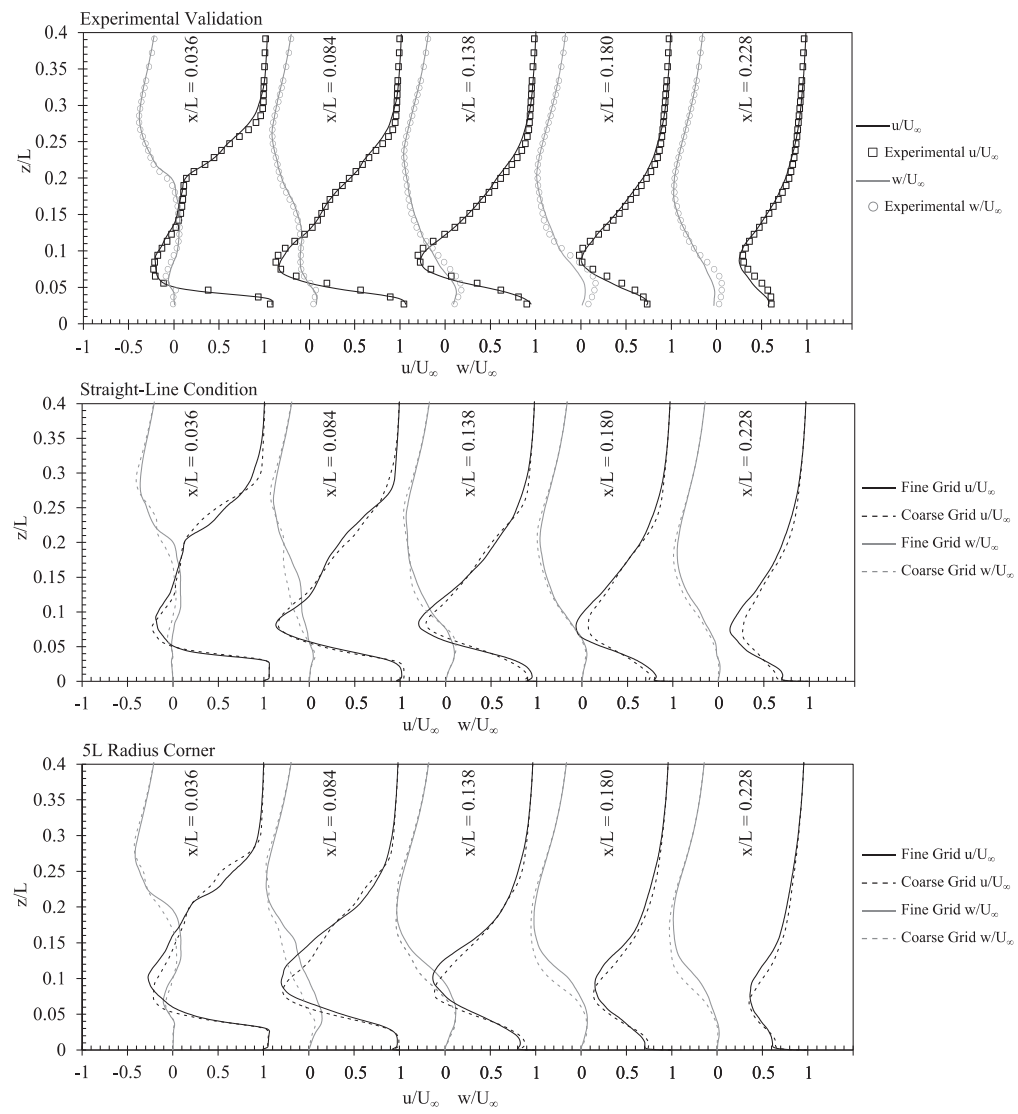

Fig. 4. Downstream profiles of $x$ and $z$-velocity components: (a) Compared to the experimental data of Lienhart and Becker (2003), and for coarse and fine grid solutions for the (b) straight-line condition, and (c) $5 \mathrm{~L}$ radius cornering condition.

(Krajnović and Davidson, 2005b; Thacker et al., 2012). In this instance a suitable solution averaging time, $\frac{t U_{\infty}}{L}$, was found to be 10 $\left(1 \times 10^{5}\right.$ time-steps) - further averaging time yielded differences that were proportionally minor for the flow effects presented. This was determined through monitoring of forces and the change in velocity and pressure distribution on downstream planes throughout the averaging period. The solution was initialized from a RANS solution with a Realizable $k$-epsilon turbulence model, followed by a series of initial time-steps. The fully developed flowfield state can only truly be analysed retrospectively. The aforementioned varying time scales of transient flow behaviour further complicated this assessment. Frequency analyses of 18 different points, located in the near wake and upper boundary layer, identified a number of separate peaks in the spectrum for any given location - contributing to the lack of easily recognisable periodic behaviour. A similar methodology was adopted to that of prior analyses (Krajnović and Davidson, 2005a), where point velocity measurements were taken both around the body and downstream for each time-step. For two successive passages of the flow over the body, it was ensured the median velocity magnitude values fell within $0.5 \mathrm{~m} / \mathrm{s}$ at measured locations. It was determined that a minimum of $4 \times 10^{4}$ time-steps was required to achieve the initial developed state, and this period was used for all cases.

\section{Validation and verification of numerical model}

Wake profiles of both $x$ and $z$-velocity components along the symmetry plane were taken on several downstream locations and compared to experimental LDA results, as is shown in Fig. 4. The fine grid solution, which adopted boundary conditions as the experimental configuration of Lienhart and Becker (2003), is shown in

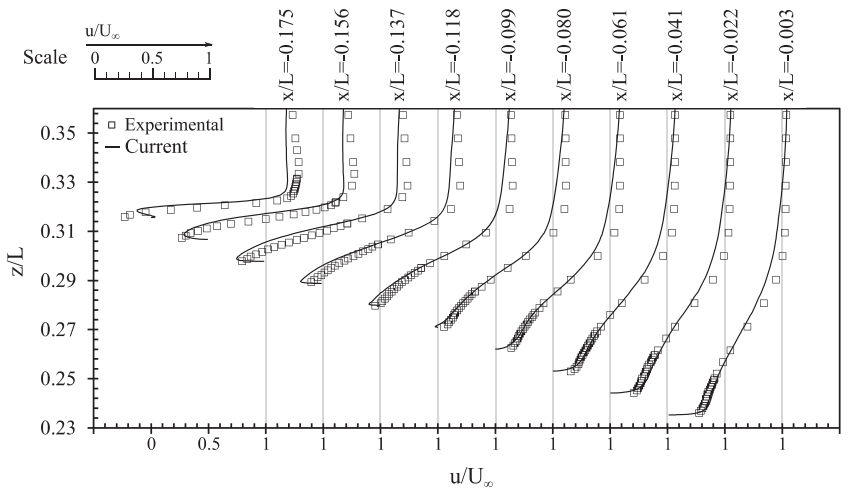

Fig. 5. Profiles of normalised $x$-velocity over the backlight surface compared to the experimental results of Lienhart and Becker (2003).

Fig. 4a. These results indicated that both the time-averaged wake deficit and the wake dissipation were closely captured by the numerical model. Profiles of the vertical velocity component ensured the flow angles off the backlight and the lower surfaces were also accurately modelled. A noted discrepancy was the underprediction of the vertical velocity component within the lower part of the wake, as evident on profiles $x / L=0.132-0.228$. In conjunction with a more significant $x$-velocity deficit at $x / L=0.228$ in the numerical model, this would suggest an over-prediction of the wake separation bubble. Despite this discrepancy the largely favourable agreement with the experimental results demonstrated the ability of the numerical model to capture the flow characteristics along the symmetry plane, however further analysis of the entire wake structure was required to assess the validity outside this plane. 
a

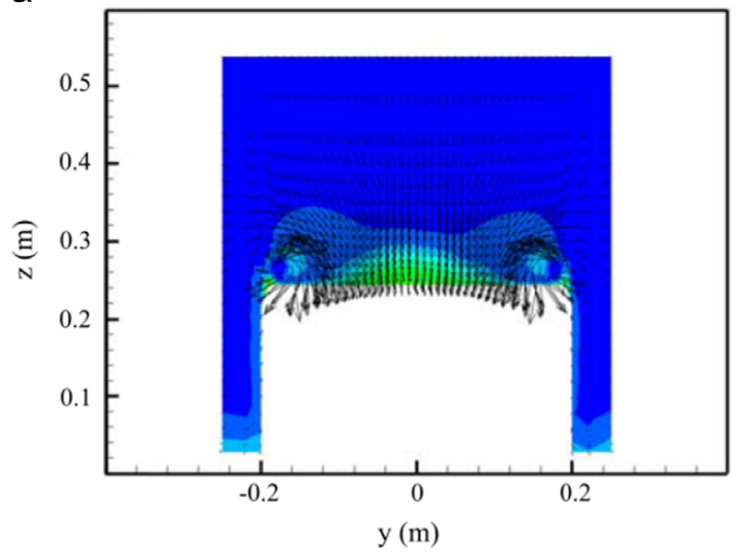

C

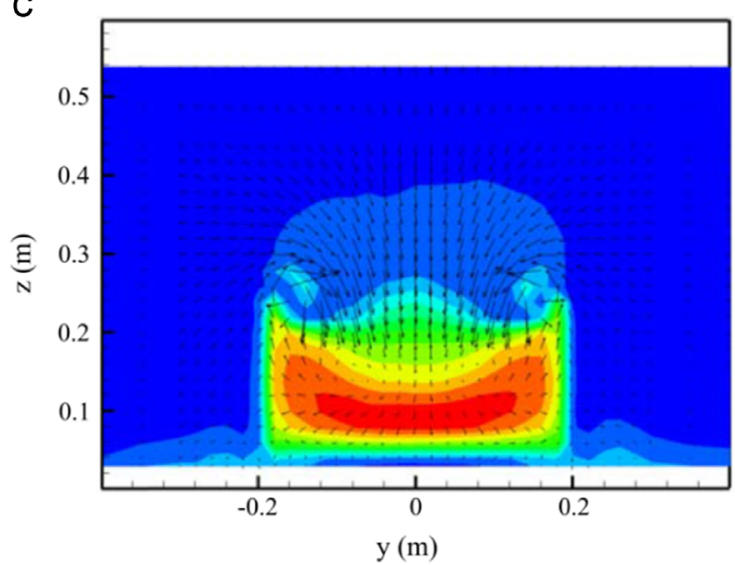

e

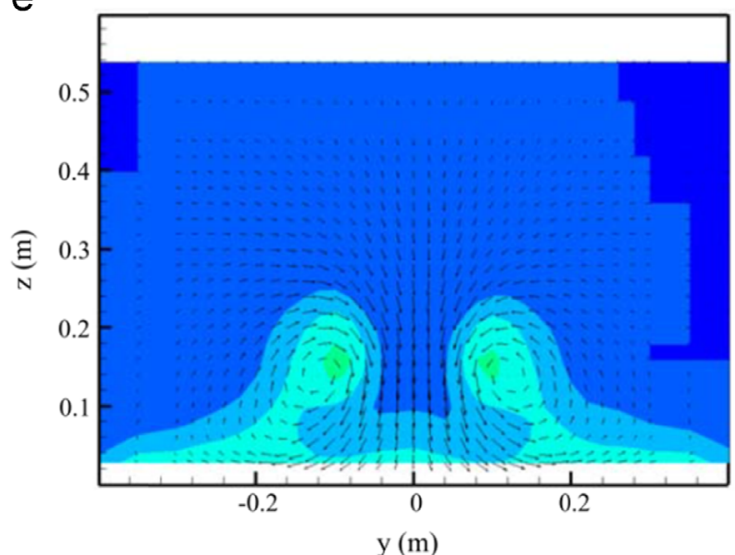

b

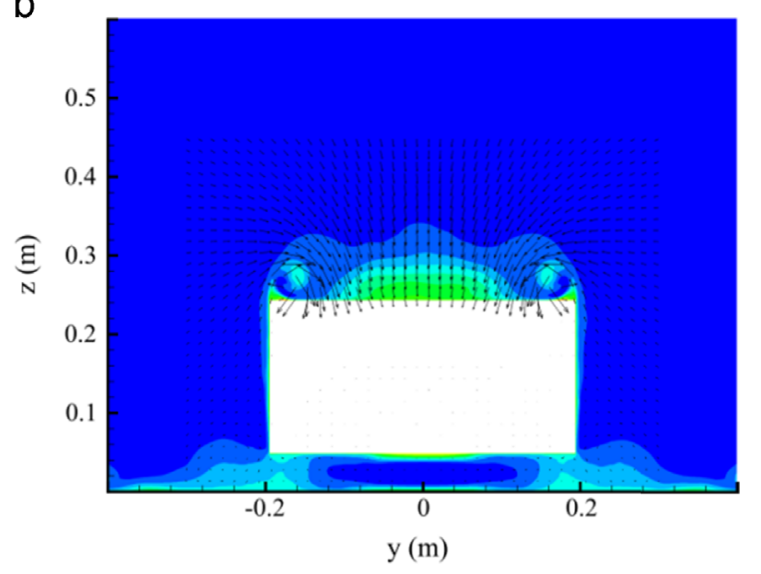

d

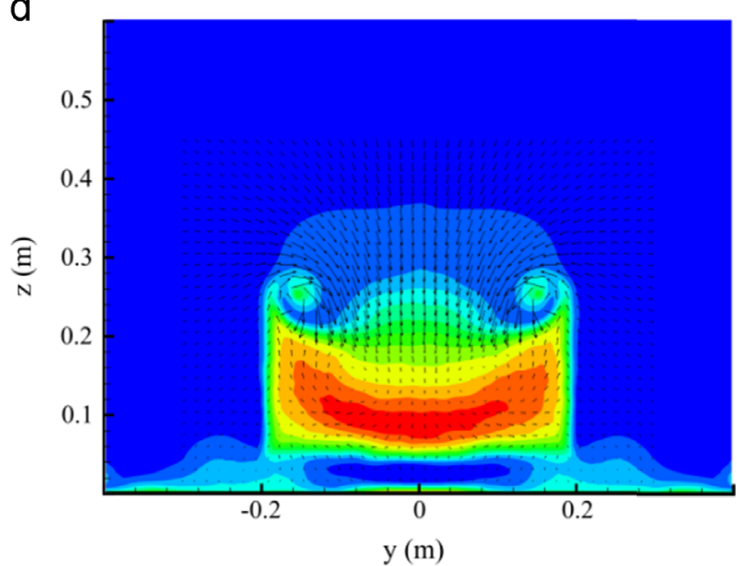

$f$

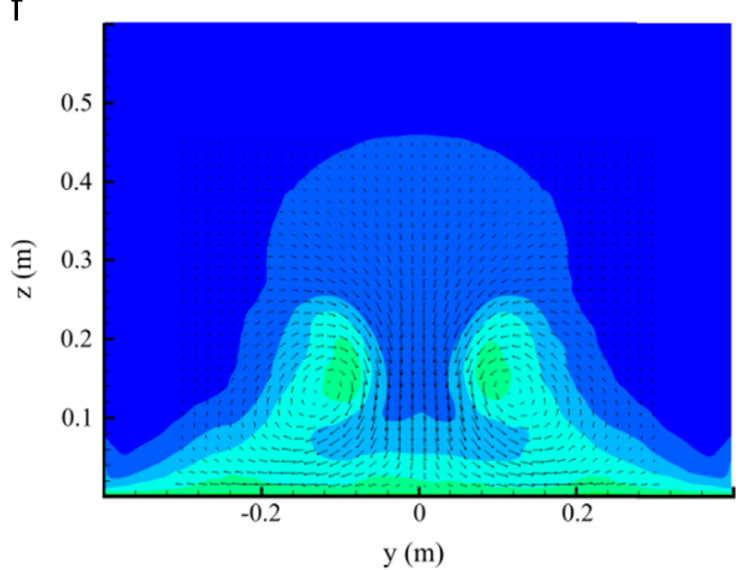

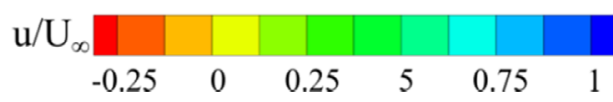

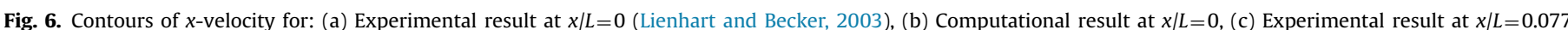

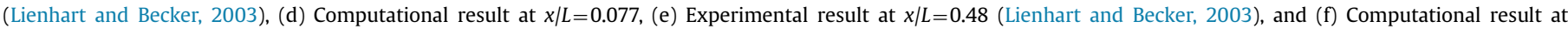
$x / L=0.48$.

The prediction of the central near wake was also analysed for the straight-line condition in the absence of the supporting struts, and the smallest radius cornering condition which was a 5 length radius. These profiles are shown in Fig. $4 \mathrm{~b}$ and $\mathrm{c}$ where the fine grid result $\left(30 \times 10^{6}\right.$ cells $)$ was compared to a coarser grid $\left(11 \times 10^{6}\right)$ with the same adopted meshing strategy. Initial differences in the wake structure between the cornering condition and the straight-line condition become apparent, and these effects are discussed in further detail throughout Section 4. In both instances the profiles taken for the coarse grid indicated a comparatively reduced velocity deficit in the wake region, which could be attributed to the expected numerical dissipation of wake structures. In the case of the $5 \mathrm{~L}$ radius cornering case this was coupled with an increase downwash angle of the flow leaving the backlight surface, as indicated by the reduced $z$-velocity component. While the comparison demonstrated grid-independent qualitative 

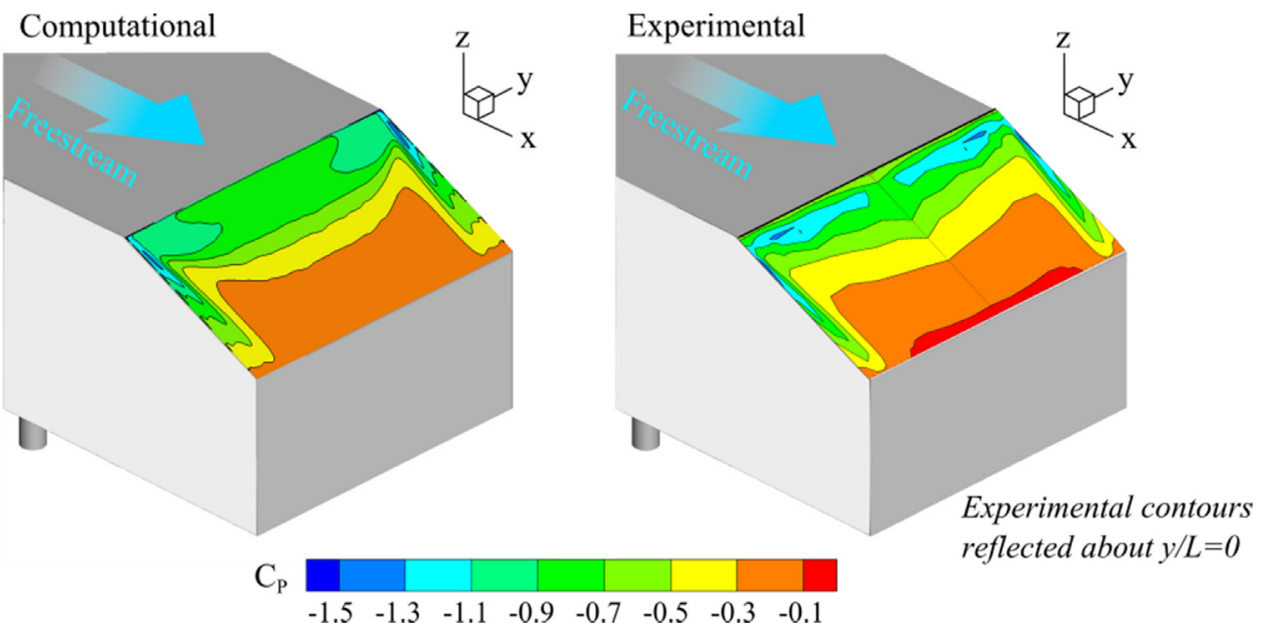

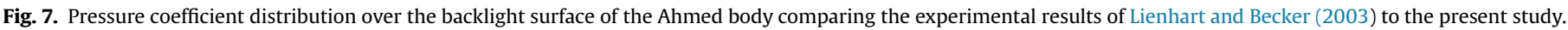

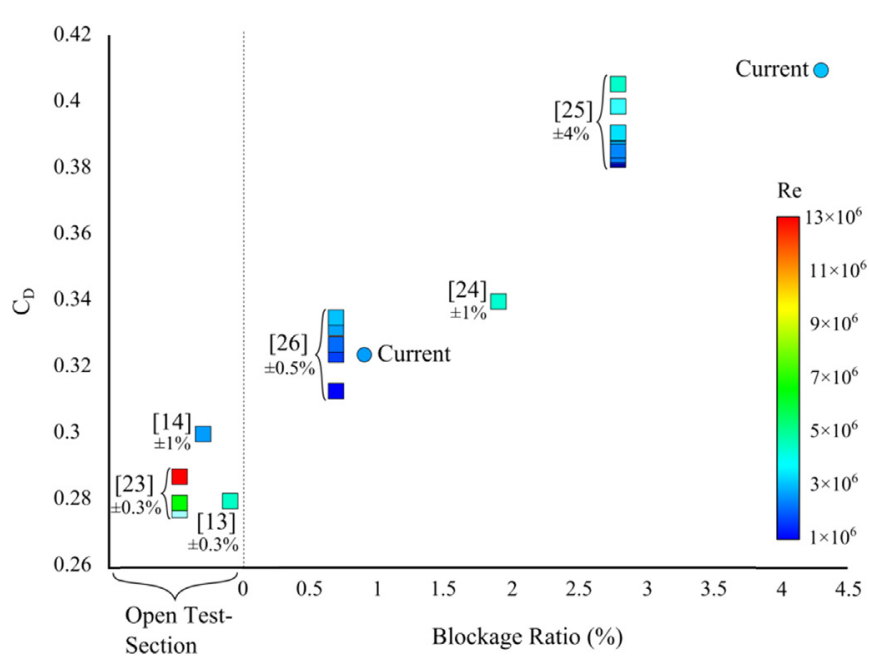

Fig. 8. Coefficient of drag comparison between present study, and past experimental results with reported maximum experimental error.

similarity, the fine grid resolution was favoured for all cases due to its preferential suitability for the numerical method.

To also ensure the separation and reattachment over the backlight surface was modelled accurately velocity profiles were compared over the backlight surface between the experimental results and the present study, as shown in Fig. 5. Profile characteristics matched favourably with the deficit and reversed flow regions within the separation bubble. It must be noted that the experimental results did not extend completely to the surface and hence it can be observed that the numerical results extend beyond the experimental data. The discrepancy within the upper region, where the numerical model predicted a lower velocity in comparison to experimental results, was attributed to an overprediction of the forebody separation bubble. This resulted in an increased energy deficit over the upper surface. The cumulative effect resulted in a more significant deficit extending the length of the backlight due to the corresponding loss.

Downstream velocity contours were also compared to the timeaveraged experimental results of Lienhart and Becker (2003), as is shown in Fig. 6. The shape and position of the dominant C-pillar vortices was captured along with the velocity deficit in the core. A point of difference was the shear layer structure shed from underneath the model where experimentally, a more even layer was observed. In the numerical model the higher criticality of the backlight, edges, and the forebody dictated the assignment of a high cell concentration in these regions, which was at the expense of mesh resolution in regions such as the wake of the struts. While the wake structure of the struts was captured, the cell growth rate from the surface numerically diffused this region. These struts were not present for any further cases, so this was of lower importance than accurately capturing the flow over the backlight and upper surfaces of the body.

An additional survey of static pressure coefficient over the rear of the body was also undertaken in the experiments and this was compared to the numerical simulations, as is shown in Fig. 7. The numerical results predict both the presence of key flow features and their location, although the experimental resolution was lower. The low pressure regions caused by the C-pillar vortices extending down either side of the surface in both results, as well as the low pressure region beneath the separation bubble. The reattachment length indicated in the experimental results appears to occur further downstream; however a further quantitative assessment would require direct comparison of wall shear which was not included in the experimental study.

Measurement of the drag acting on the body was not part of Lienhart and Becker (2003) study. Whereas, the experimental study of Joseph et al. (2012) included drag measurements in addition to the effect of Reynolds number on drag coefficient. They used a closed test-section with a blockage ratio of $0.7 \%$, and this allowed comparison with the present numerical results for the straight-line configuration. Fig. 8 compares a range of reported experimental drag coefficients to the present work, with maximum reported experimental error shown next to the data. All studies used a forcebalance to attain the values shown. The experiments of Thacker et al. (2012) and Conan et al. (2011) were conducted at blockage ratios of $2.8 \%$ and $1.9 \%$ respectively, and Fig. 8 indicates that their reported drag values were respectively $36.7 \%$ and $19.4 \%$ higher than the average coefficient of 0.285 for open test section experiments. Potentially this trend across studies could indicate a greater dependency on blockage ratio than is suggested by approximate methods such as Maskell theory (Maskell, 1963) which anticipates only a $1.7 \%$ increase in drag at a blockage ratio of $1.9 \%$ (at $\left.R e=4.29 \times 10^{6}\right)$ (Conan et al., 2011). Particularly in the case of prior numerical studies at the $25^{\circ}$ backlight angle, they have tended to over-predict the drag coefficient; however the blockage ratio is seldom identified as a potential reason (Krajnović and Davidson, 2004, 2005a, 2005b; Wang et al., 2013; Guilmineau et al., 2011). The present validation and straight-line numerical cases both predict drag coefficients that are in agreement with prior experimental results. The calculated drag coefficient from the straight-line numerical simulation is 3.0\% lower than the experimental result of 


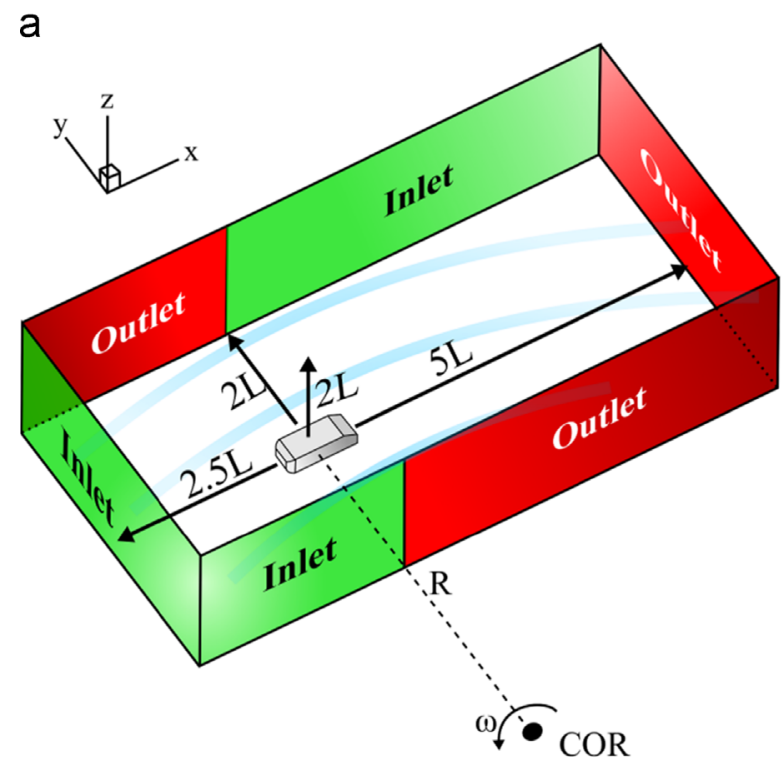

b
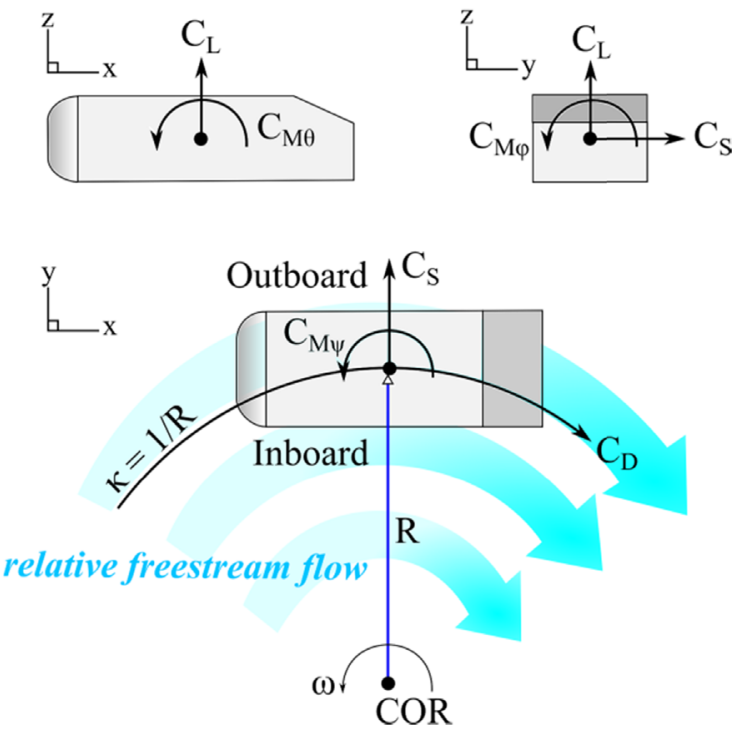

Fig. 9. Boundary conditions and extents of the computational domain (not to scale) used in the present investigation.

Table 1

Corner radii analysed in the present study.

\begin{tabular}{llcl}
\hline Radius $(L)$ & Curvature $\left(L^{-1}\right)$ & $\Psi_{1}\left({ }^{\circ}\right)$ & $\Psi_{2}\left(^{\circ}\right)$ \\
\hline Straight-line $(\infty)$ & 0 & 0 & 0 \\
20 & 0.05 & -1.43 & 1.43 \\
10 & 0.1 & -2.86 & 2.86 \\
5 & 0.2 & -5.71 & 5.71 \\
\hline
\end{tabular}

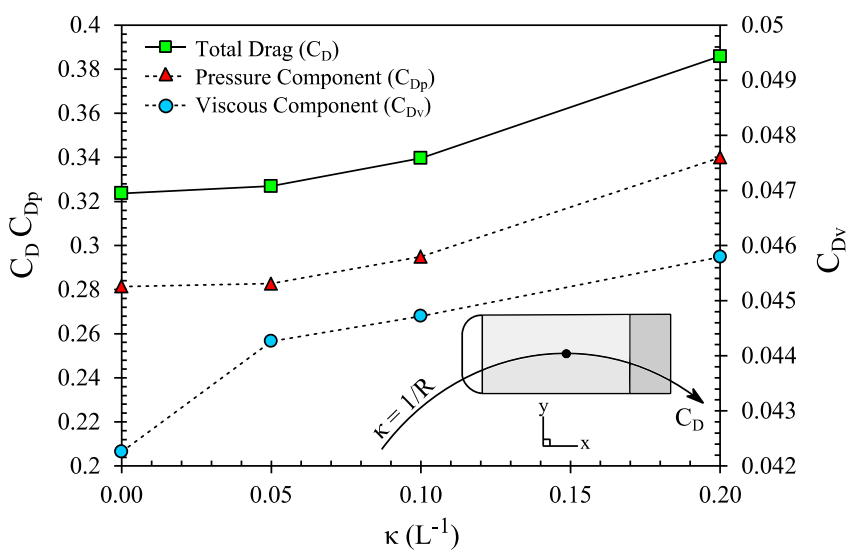

Fig. 10. Plot of aerodynamic drag coefficient against curvature of the vehicle's path.

Joseph et al. (2012) at the same Reynolds number. (Krajnović and Davidson, 2005c) investigated the influence of ground plane motion for the same geometry and identified an increase in spanwise mass flux which led to an increase in drag for the case with a stationary ground. As this experiment did not include a moving ground, this explanation would likely accommodate the observed discrepancy.

The validation of the computational model demonstrates the ability of Wall-Resolved LES to capture the flow structures developing in the wake of the Ahmed reference model, particularly the dominant C-pillar vortex and the separation bubble on the rear backlight surface. Furthermore, results demonstrated that the drag coefficient was also predicted within the expected range in comparison to prior experimental results.

\section{The steady-state cornering condition}

Modelling a vehicle in the cornering condition requires a noninertial reference frame, and this necessitates the introduction of relative velocity and acceleration terms to accommodate for the flow behaviour within a rotating reference frame. In the present study this increased solution time by approximately $50 \%$ when solving for a rotating reference frame.

Boundary conditions and the structure of the domain have differed amongst previous cornering studies (Tsubokura et al., 2012; Okada et al., Fujita; Nara et al., 2014; Keogh et al., 2015a, 2015b). In this study, only steady-state constant radius corners are considered and this was most efficiently achieved through the use of a single rotating reference frame applied to the entire domain. Motion was defined by prescribing an angular velocity about a point external to the domain where the distance of this point from the body is equal to the corner radius.

The use of a rectangular domain with modified inlet and outlet locations has been favoured in prior analyses (Tsubokura et al., 2012; Okada et al., Fujita; Nara et al., 2014; Keogh et al., 2015b), and is shown in Fig. 9a. A favourable attribute is that it permits the evaluation of multiple types of condition utilising the same grid. This allows a reduction in the overall time required to achieve multiple results and would also permit dynamic simulations incorporating variable curvatures.

\subsection{Aerodynamic evaluation in the cornering condition}

Aerodynamic drag is the resistance due to the flow in the direction of a body's motion. Therefore as a vehicle travels in the curved path of a corner, drag itself also acts in a curved path (Keogh et al., 2015a, 2015b) as is shown in Fig. 9b). Drag becomes proportional to the moment acting about the centre of rotation of the vehicle's path. The direction of lift remains unaffected as it is in the vertical direction, parallel to the axis of rotation. Side force will also continue to act in the same direction, but will differ slightly from the radial force. All moments will continue to act in the same direction. For the complete derivation of these coefficients please refer to Keogh et al. (2015b). 


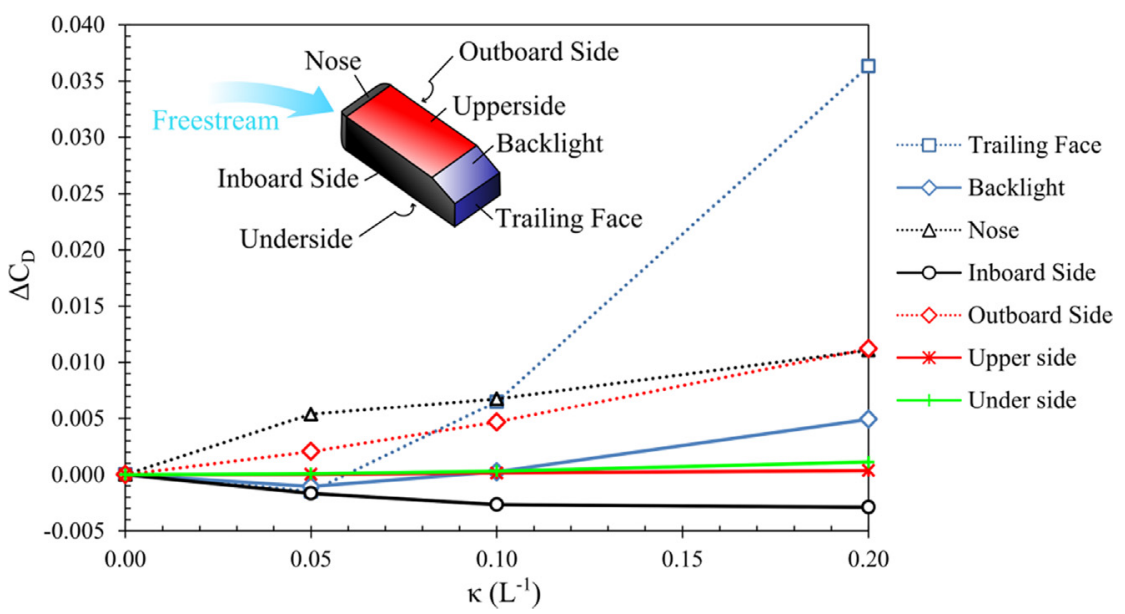

Fig. 11. Change in overall drag coefficient contribution for individual surfaces of the body due to decrease in corner radius.

\section{Results}

\subsection{Aerodynamic forces and moments}

The curvature of a vehicle's path through a corner is inversely proportional to the radius, and all cornering-specific flow effects can be seen to increase as curvature also increases. The corner radii investigated are representative of those that would commonly be observed in real-world scenarios. The radii, and the front and rear flow angles they correspond to, are shown in Table 1.

There is an increase in the aerodynamic drag coefficient with a decrease in corner radius - this followed an exponential trend as shown in Fig. 10. The drag coefficient was $19.2 \%$ greater for the $5 L$ radius corner when compared to the straight-line condition, and respectively less for larger radii corners.

The proportional contribution of the viscous component to the total drag coefficient was a maximum of $13.5 \%$, occurring in the $20 \mathrm{~L}$ radius cornering case. These values were slightly below the $15 \%$ contribution reported in the initial findings of Ahmed et al. (1984). The maximum percentage increase in the magnitude of the viscous drag value was $8.3 \%$ and occurred for the $5 \mathrm{~L}$ radius corner case, due to an increase in the outboard-side boundary-layer thickness, which is discussed in more detail in further sections. Largely the increase in drag was attributed to the more dominant pressure component. This was similar to the findings in prior research of the straight-line condition, where the near wake flowstructure was the most influential region.

Fig. 11 details the change in drag coefficient contribution over each surface, with the cornering cases compared to the straightline results. The trailing face of the body can be identified as having the greatest effect toward the overall increase in drag. The increased pressure deficit at the rear of the body, due to the change in wake structure, resulted in decreased pressure over the surface. The increase in drag over the outboard side and decrease for the inboard side occurred due to the disparity in boundary layer growth and the flow which occurred at the rear of the body which caused an increase in the local viscous component, as discussed further in Section 5.2.

Change in both flow structure and pressure distribution over the body also resulted in the introduction of an aerodynamic side force and yawing moment. Both occurred primarily due to the resultant surface pressure distribution over the sides of the body. Lift coefficient did not follow a consistent trend with corner radius, and the maximum reduction was $3.9 \%$ for the $5 \mathrm{~L}$ radius corner, shown in Fig. 12.

The high pressure on the outboard side and lower pressure on the inboard resulted in a negative side force acting towards the

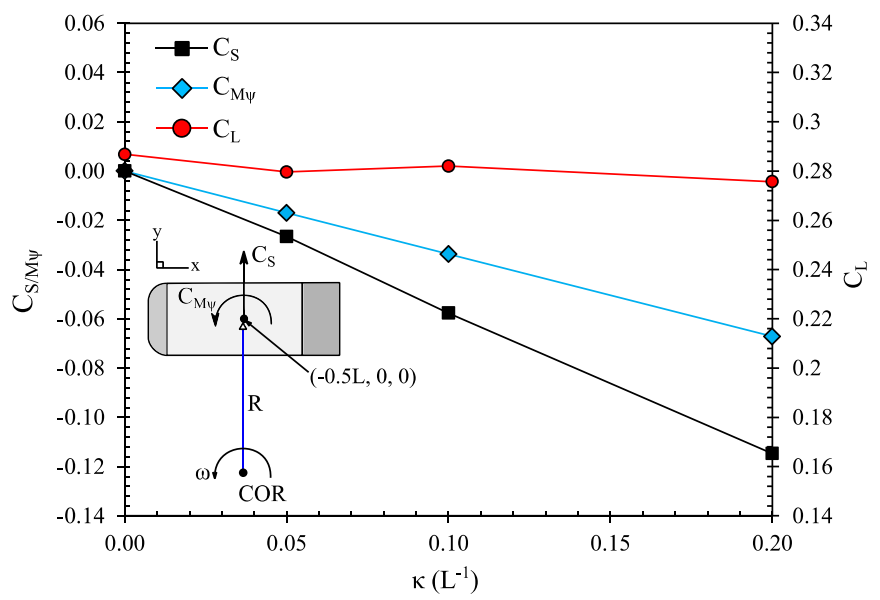

Fig. 12. Lift coefficient, Yawing moment coefficient and side force coefficient for all cases.

centre of rotation. The magnitude of this force followed a nearlinear trend as the curvature of the path increased. A yawing moment occurred due to a rearward centre of pressure over the outboard side where a linear trend was again observed for the radii considered and the maximum coefficient was -0.07 .

\subsection{Longitudinal flow structure}

For the cornering cases, as the corner radius decreased the near wake structure became more asymmetric. The outboard flow structures became more dominant in the wake region, particularly due to an increase in strength of the outboard C-pillar vortex. While the body itself had a straightening effect on the flow (in terms of alignment with the $x$-axis) the wake tended toward the direction of the freestream flow.

The asymmetry in the wake region occurred due to the change in the way the bluff body was perceived by the freestream flow. With the freestream flow no longer travelling in a straight-line the flow assumes a variable angle of yaw along the length of the body, and across the width (to a smaller extent).

Isosurfaces of instantaneous $Q$-criterion demonstrated the highly turbulent nature of the flowfield. The mean location of the two C-pillar vortices can be identified using time-averaged $x$-vorticity in Fig. 13. For the instantaneous flowfield there was a clear difference in the wake region and the path that flow structures followed downstream. What was not apparent in the timeaveraged flowfield was the interaction occurring between flow 


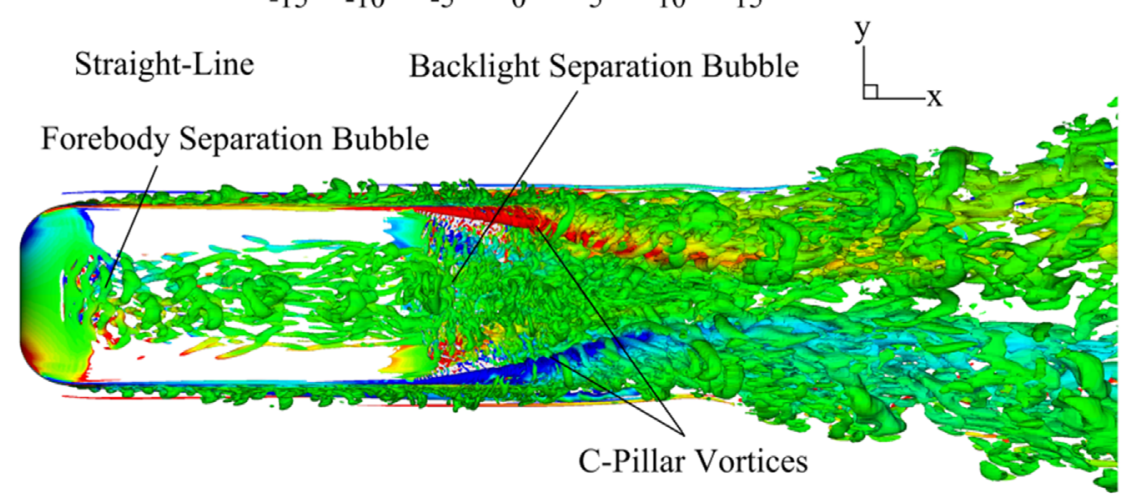

b 10L Radius Corner $\quad$ t

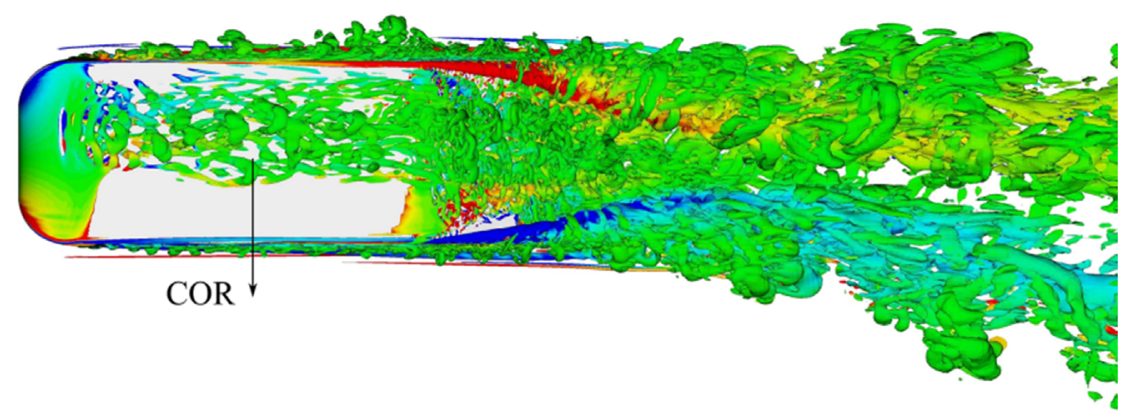

Fig. 13. Normalised instantaneous Q-criterion isosurfaces of 34.9 with contours of time-averaged normalised $x$-vorticity for (a) the straight-line condition, and (b) $10 L$ radius cornering condition.

a
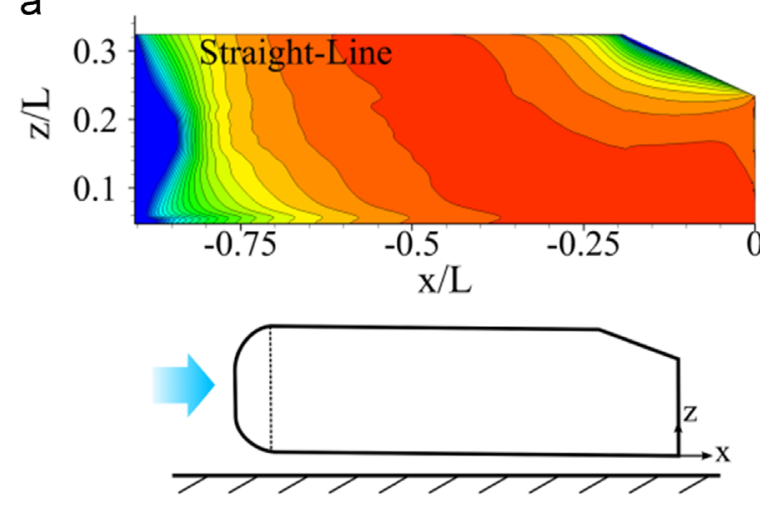

$\mathrm{C}_{\mathrm{P}}$

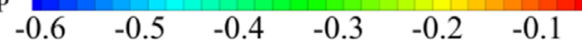

b
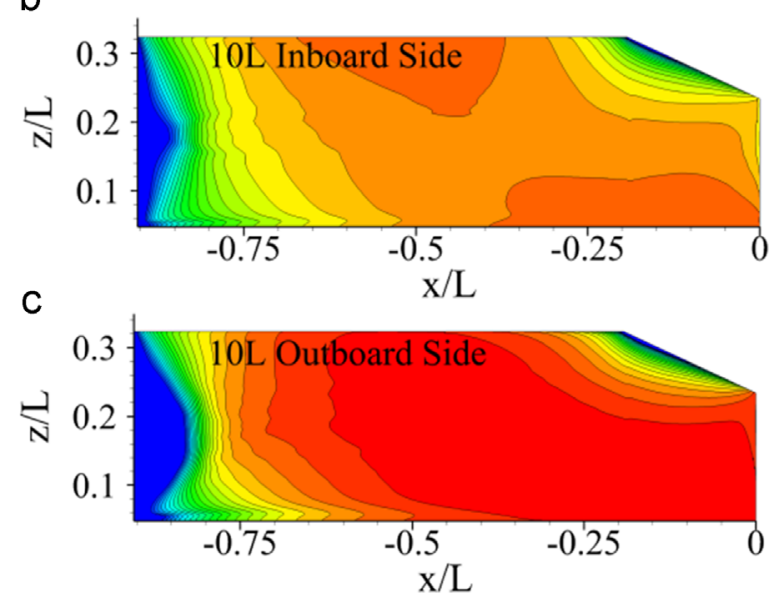

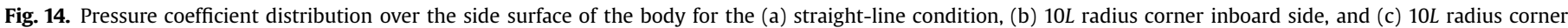
outboard side.

structures at the front and rear. The separation bubble aft of the nose, which was investigated in detail by Krajnović and Davidson (2005b), resulted in hairpin vortices which propagated downstream to interact with flow over the backlight.

Due to the change in perception of the body by the freestream flow, the flat sides of the bluff body acted according to their perceived curvature. Due to this the inboard side was perceived as a convexly curved surface and caused a rearward local expansion which aided in acceleration of the flow. The outboard side behaved in the opposite manner and affected a local rearward deceleration and increase in pressure. These effects were coupled with a radially orientated positive pressure gradient within the boundary layer due to the rotating non-inertial reference frame where the combined effects resulted in a decrease in pressure on the inboard side and an outboard increase. This can be observed in Fig. 14 noting that the disparity was most significant near the trailing face.

Due to the increased flow angle past the outboard side of the nose a thicker boundary layer developed over the outboard side. In conjunction with the rearward change in flow angle, increased shear was responsible for a local increase in viscous drag. A thinner boundary layer develops over the inboard side, relative to the 
a

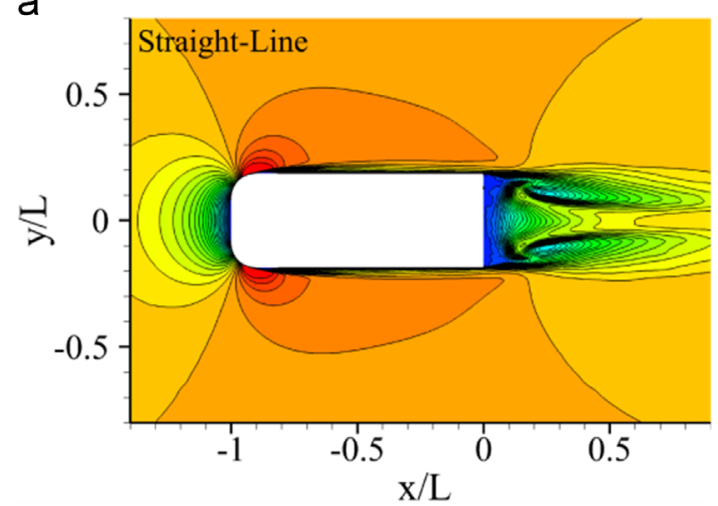

C

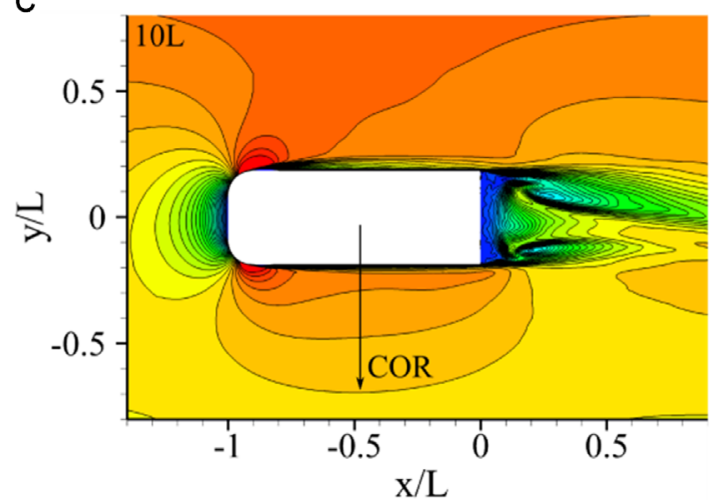

b

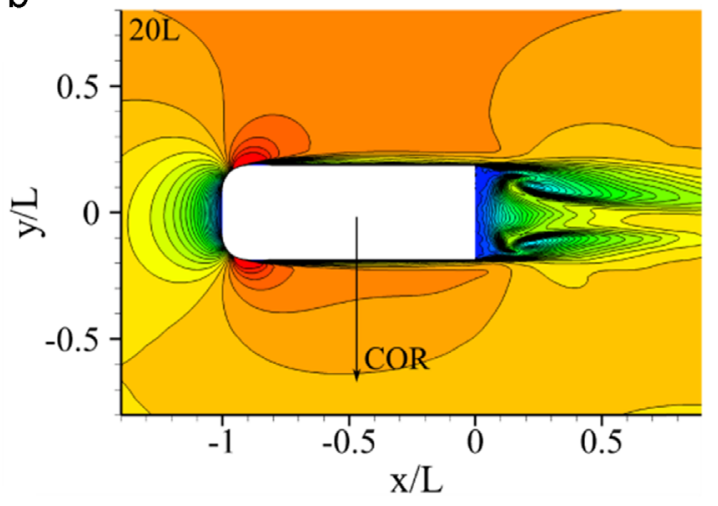

d

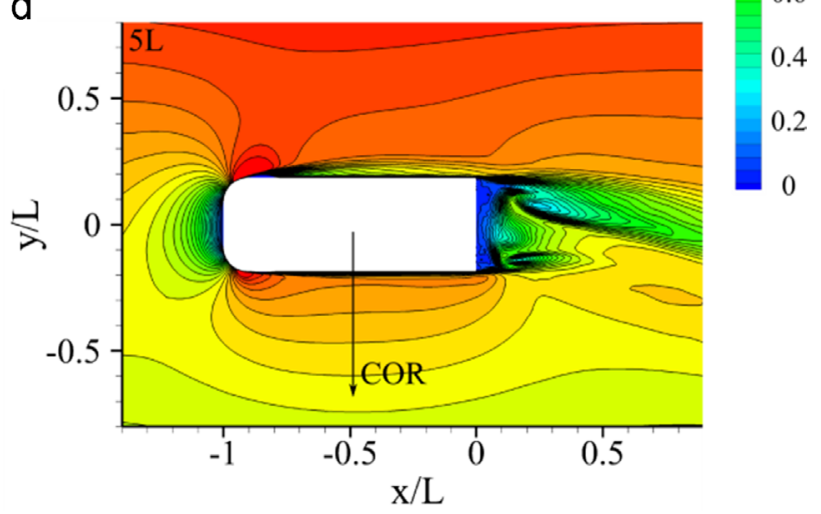

Fig. 15. $X$-velocity contours at $z / L=0.18$ for (a) the straight-line case, (b) $20 L$ radius corner, (c) $10 L$ radius corner, and (d) $5 L$ radius corner.

a

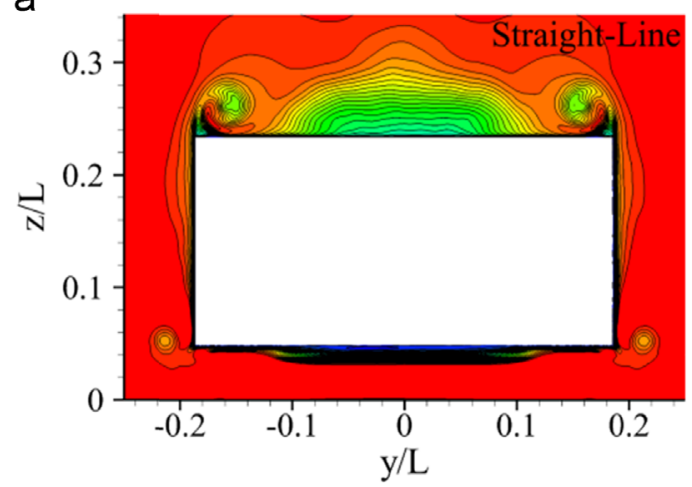

C

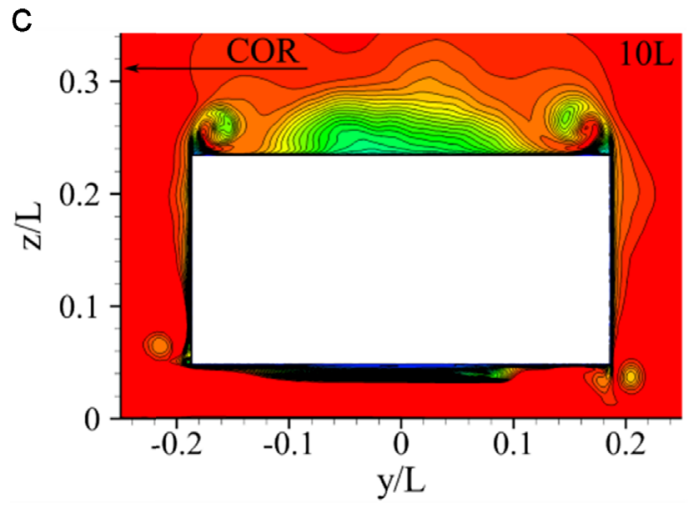

b

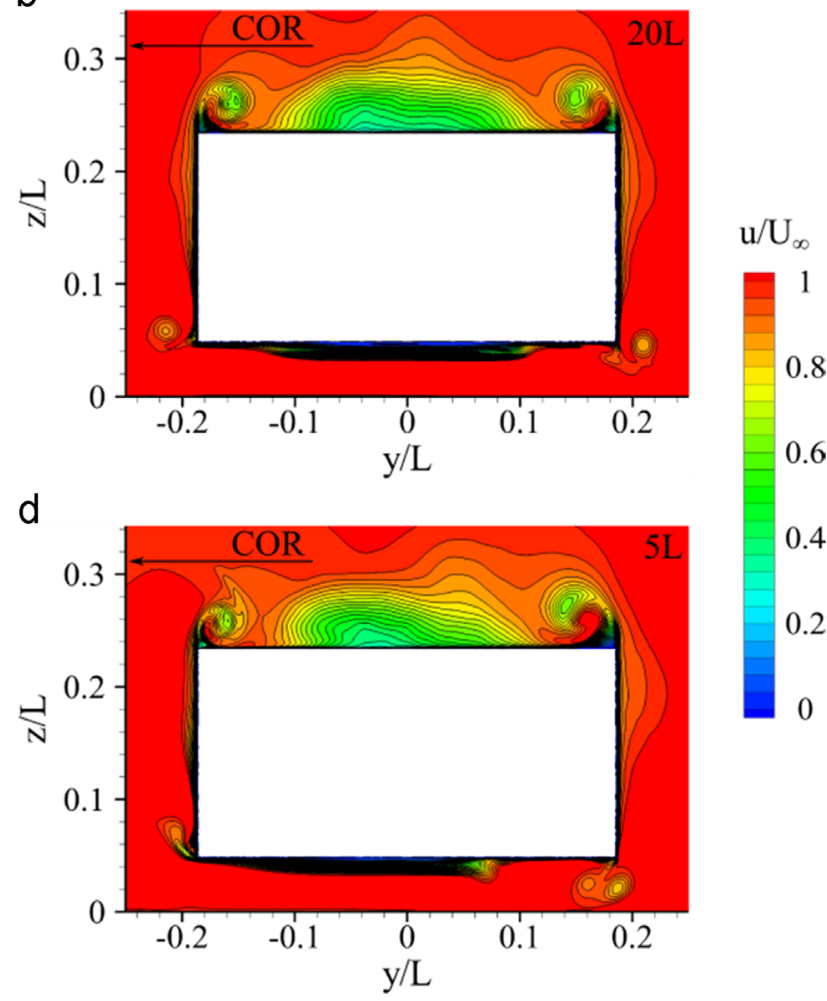

Fig. 16. $X$-velocity contours at $x / L=0$ showing the wake configuration at the trailing face of the body.

straight-line condition, and a small local viscous drag reduction. This disparity became more evident in the small radius corners - visible in Fig. $15 \mathrm{c}$ and d. For the $5 \mathrm{~L}$ cornering case the outboard boundary layer measured at the centre of the face $(z / L=0.18, \quad x / L=-0.5)$ was $54.1 \%$ thicker than the respective inboard location. 
These flow effects occurring over the sides of the body then affected the mechanisms responsible for the formation of the Cpillar vortex, which can be observed in Fig. 15. In the straight-line condition this vortex forms when the shear layer from the side rolls up and is drawn to the low-pressure region over the backlight (Ahmed et al., 1984), similar to the flow mechanism for a wing (Diasinos et al., 2013), and this remained identical in the cornering condition.

The increased pressure on the outboard side of the body increased the gradient across the C-pillar location and resulted in the formation of a larger vortex, shown at the trailing face in Fig. 16. As the curvature of the body's path decreased the vortices assumed a symmetrical configuration similar to the straight-line condition.

The lower longitudinal vortices were also affected by the cornering condition. In the straight-line condition these formed due to a pressure gradient caused by an increase in pressure underneath the body and cause the vortex to form outside the body. In the cornering condition the increased pressure over the outboard side caused the pressure gradient to reverse - resulting in an additional vortex. This additional counter-rotating vortex formed underneath the body, as shown in Fig. 17. It also interacted with

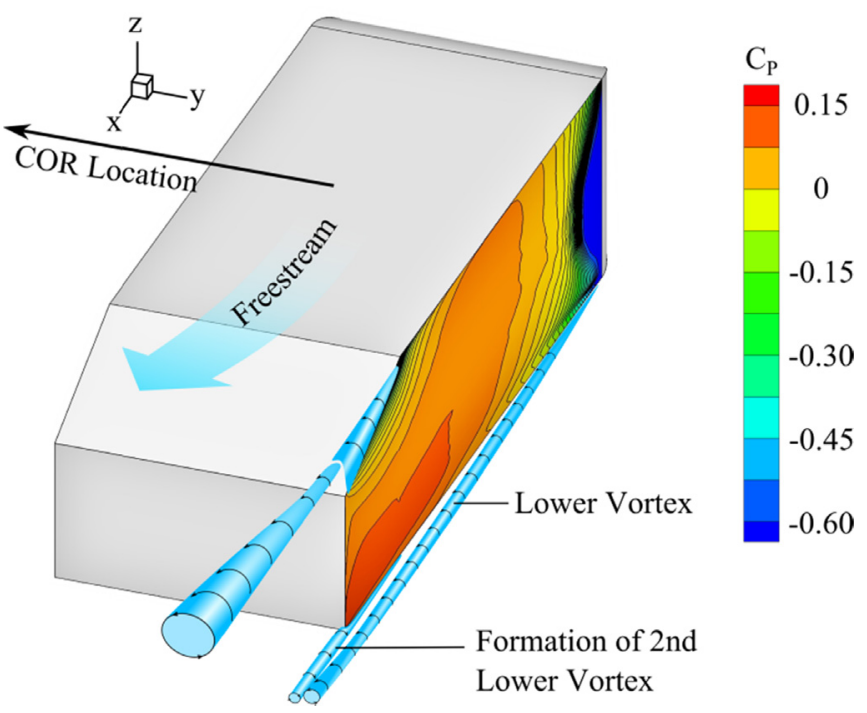

Fig. 17. Diagrammatic representation of the formation of the second lower vortex.

a
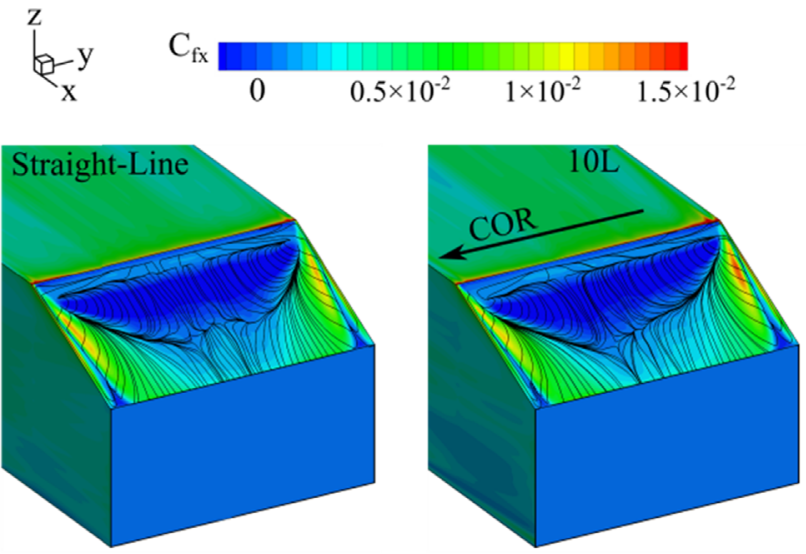

b

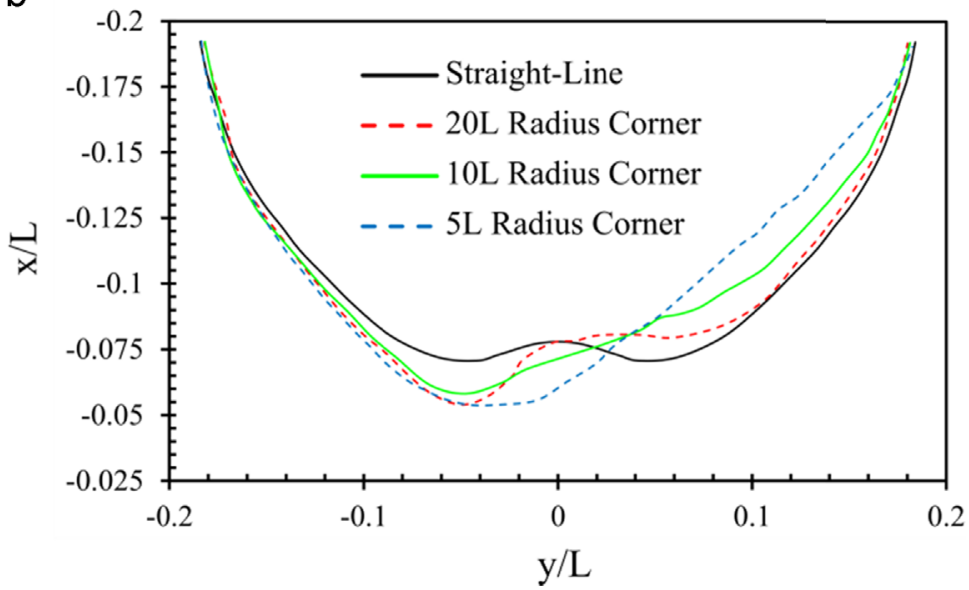

the shear layer shedding from underneath the body - displacing it further to the inboard side.

On the inboard side the lower vortex increased its displacement above the ground plane, shown in Fig. 16c and d, was drawn more towards the low pressure region. In the $5 \mathrm{~L}$ radius corner case a stretched shape was observed at the trailing face due to this interaction.

\subsection{Spanwise flow structure}

In the straight-line condition, shown in Fig. 18, the wall-shear contours and surface streaklines over the backlight show the Cpillar vortices resulted in symmetrical reattachment points of the flow on both sides of the face. The separation bubble extended furthest towards the centre of the face, reducing in length with proximity to the vortex. Fig. 18a shows the difference between the $10 L$ radius cornering case and the straight-line case. In this instance the outboard C-pillar vortex decreased the distance for reattachment of the flow on the outboard side. The decreased pressure of the vortex draws flow across the face resulted in an $18.2 \%$ wider outboard recirculation region within the separation bubble. The reattachment length increased inboard but remained similar for all cornering cases, while the width of the circulation region decreased.

The separation bubble at the rear of the body consists of two large spanwise vortices that form at the trailing face. The flow structure at $y / L=0.1$ is shown in Fig. 19a for the straight-line case. Due to the symmetry of the body, the same structure was also observed at $y / L=-0.1$ but is not shown. For the $10 L$ radius corner case the outboard C-pillar vortex and the angle of the flow across the rear of the body contributed to an elongation of the separation bubble by $4.0 \%$ on the outboard side (measured at $y / L=0.1$ ) and a decrease in the downwash angle, shown in Fig. 19b. Also observable was the effect of the increased angle of the C-pillar vortex across the backlight face; this increased the vertical extension of the pressure deficit. In the opposite manner a contraction of $13.4 \%$ occurred inboard, shown in Fig. 19c.

As a result of the contraction of the separation bubble the inboard downwash angle became steeper and the inboard C-pillar vortex followed a more downwards path. Due to this path the vortex interacted with the ground a short distance downstream, shown in Fig. 20d, and changed shape.

The outboard flow effects caused the outboard C-pillar vortex to have an increased vertical displacement due to both the extension of this separation bubble and a thicker shear layer being

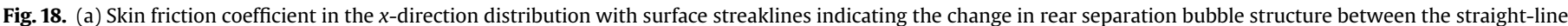
condition and $10 \mathrm{~L}$ radius corner, and (b) mean reattachment line over the backlight surface for all cases. 

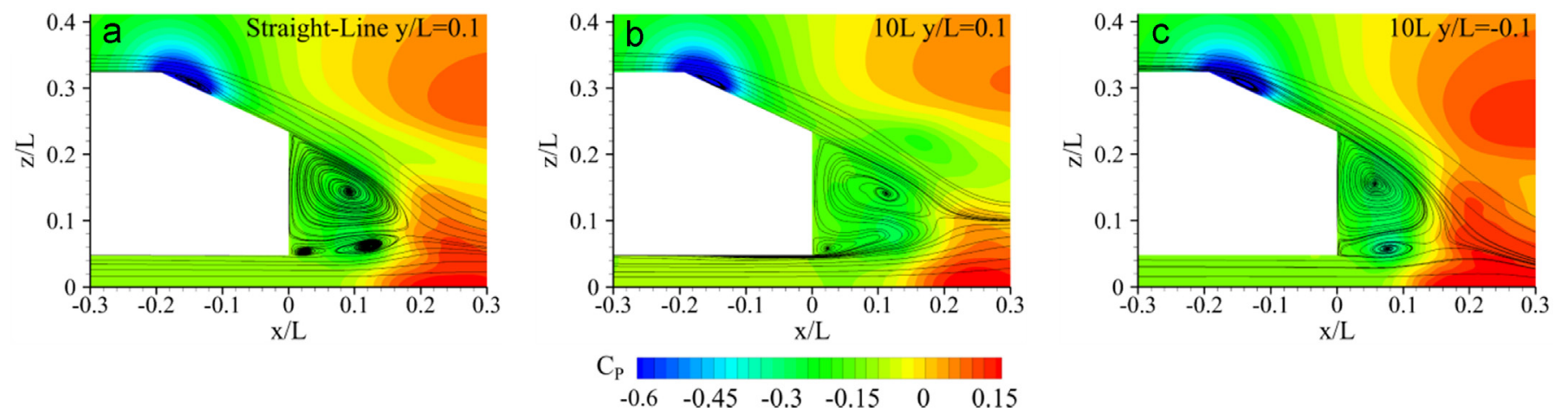

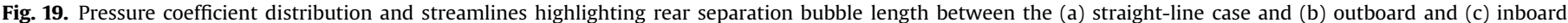
locations for the $10 \mathrm{~L}$ radius corner.
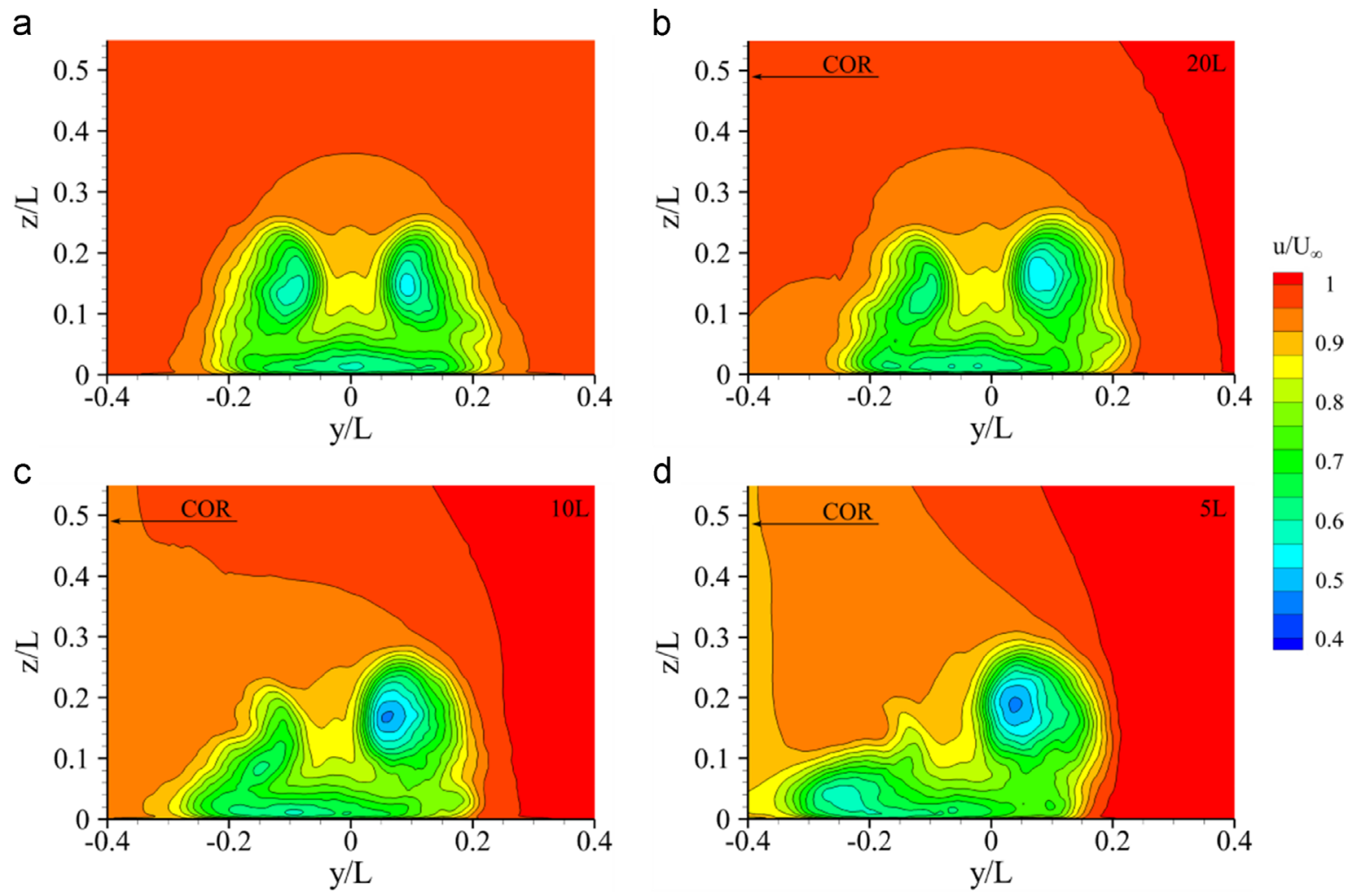

Fig. 20. Downstream wake structure shown using $x$-velocity contours at $x / L=0.48$.

drawn inwards due to the wake deficit. These changes further exacerbated the disparity in vortex size, and caused a difference in the downstream wake structure.

\section{Further discussion}

The specific attitude at which any vehicle travels through a corner will be dependent on vehicle dynamics, the speed and the condition of the road surface. For specific vehicle geometries these subtle changes would need to be accounted for and would result in certain effects becoming more or less significant.

The increase in drag coefficient was primarily attributed to the decrease in pressure over the trailing face. This, in turn, was due to an increase in the pressure and velocity deficit in the near-wake region. The asymmetric C-pillar vortex structure resulted in the delayed reattachment of the flow down the backlight angle on the inboard side and decreased the length of the separation bubble outboard. Additionally the change in direction of the hairpin vortices propagating from the upper forebody separation bubble increased the energy within the boundary layer toward the outboard side.

There was a substantial change in the downstream wake structure where a cumulative result was observed. For the smaller radii corners this resulted in the inboard vortex being in contact with the ground, while the outboard increased its vertical displacement.

From these results an interesting comparison can be made between the cornering flowfield and that identified in transient cross-wind investigations (Krajnović et al., 2012, 2011; Ferrand, 2014). In such scenarios the flow can also observe differing angles from front to rear, where a fluid particle which interacts with the front of the body will observe a different angle by the time it reaches the rear - albeit in a very different non-inertial reference frame to that of cornering. However the temporal variation between the respective flow paths (especially for flowfields 
involving separated and recirculating structures) would differ and result in a varied temporal response for flow structures. For an oscillating bluff body (Krajnović et al., 2011) identified inertial effects to result in a phase shift between the aerodynamic force and pressures, and the instantaneous condition of yaw to which they corresponded. Thus the cornering condition uniquely maintains this constant yaw variability independent of time.

Results demonstrate the potential for design modifications that could be made to mitigate the increase in aerodynamic drag, however the yawing moment and side force would be more difficult to negate. The pressure gradient across the sides of the body was identified to remain inherent to the rotating non-inertial reference frame of the cornering condition. Designs which enhance this side force would be of benefit for increasing the available lateral grip, while the negative yawing moment could be utilised to damp the yawing moment imparted on the vehicle by a driver through a corner.

\section{Conclusion}

Wall-resolved Large Eddy Simulations (LES) were able to offer detailed insight into the flow structures developing around a simplified vehicle geometry in steady-state corners. A $19.2 \%$ increase in aerodynamic drag coefficient was found to occur for a 5 car-length radius corner. The increase in drag was attributed to the change in the near-wake structure, and related exponentially to the curvature of the body's path. An asymmetric flow structure occurred around the body and resulted in a surface pressure distribution change over the body. These same changes resulted in the introduction of a yawing moment coefficient which damped the rotation of the body through a corner, and a side force which acted towards the centre of the corner. Results demonstrate the potentially high importance of evaluating the cornering condition during the aerodynamic development of vehicles.

\section{Acknowledgements}

The authors would like to acknowledge the use of the UNSW Australia Leonardi and Trentino computing clusters for the simulations conducted in the present work.

\section{References}

Ahmed, S.R., Ramm, G., Faitin, G., 1984. Some salient features of the time-averaged ground vehicle wake. SAE Technical Paper No. 840300

Barber, T.J., Leonardi, E., Archer, R.D., 2002. Causes for discrepancies in ground effect analyses. Aeronaut. J. 106 (1066), 653-657.

Bayraktar, I., Landman, D., Baysal, O., 2001. Experimental and computational investigation of Ahmed body for ground vehicle aerodynamics. SAE Technical Paper, 2001-01-2742.

Bird, B., Jaquet, L., Field, L., 1953. Effect of fuselage and tail surface on low-speed yawing characteristics of a swept-wing model as determined in curved-flow test-section of langley stability tunnel. NACA Technical Note No. 2483.

Conan, B., Anthoine, J., Planquart, P., 2011. Experimental aerodynamic study of a car-type bluff body. Exp. Fluids 50, 1273-1284. http://dx.doi.org/10.1007/ s00348-010-0992-z.

D'Hooge, A., Palin, R., Rebbeck, L., Gargoloff, J., et al., 2014. Alternative simulation methods for assessing aerodynamic drag in realistic crosswind. SAE Int. J. Passeng. Cars - Mech. Syst. 7, 617-625.
Diasinos, S., Barber, T.J., Doig, G., 2013. Influence of wing span on the aerodynamics of wings in ground effect. Proc. Inst. Mech. Eng. Part G J. Aero. Eng. 227 (3) 569-573. http://dx.doi.org/10.1177/0954410011434884.

Ferrand, V., 2014. Forces and flow structures on a simplified car model exposed to an unsteady harmonic crosswind. J. Fluids Eng. 136 (1), 011101.

Gordes, A., Process for simulating curved airflow on wheeled vehicles in fluid channels with a straight measuring section. Patent No. EP1610111A2, Germany, 2005

Guilmineau, E., Deng, G.B., Wackers, J., 2011. Numerical simulation with a DES approach for automotive flow. J. Fluids Struct. 27, 807-816.

Joseph, P., Amandolese, X., Aider, J.L., 2012. Drag reduction on the $25^{\circ}$ slant angle Ahmed reference body using pulsed jets. Exp. Fluids 52, 1169-1185. http://dx doi.org/10.1007/s00348-011-1245-5.

Katz, J., 2006. Aerodynamics of race cars. Ann. Rev. Fluid Mech. 38, 27-63. http://dx. doi.org/10.1146/annurev.fluid.38.050304.092016.

Keogh, J., Doig, G., Diasinos, S., Barber, T., 2015a. The influence of cornering on the vortical wake structures of an inverted wing. Proc IMechE Part D J. Auto. Eng. 1-13.

Keogh, J., Barber, T., Diasinos, S., Doig, G., 2015. Techniques for aerodynamic analysis of cornering vehicles. SAE Technical Paper 2015b-01-0022.

Keogh, James, Tracie Barber, Sammy Diasinos, Graham Doig, 2016. A new type of wind tunnel for the evaluation of curved motion. In: Proceedings of the 54th AIAA Aerospace Sciences Meeting, p. 1774.

Krajnović, S., Davidson, L., 2005. Influence of floor motions in wind tunnels on the aerodynamics of road vehicles. J. Wind Eng. Ind. Aerodyn. 93, 677-696.

Krajnović, S., Davidson, L., 2005a. Flow around a simplified car, Part 1: Large Eddy Simulation. J. Fluids Eng. 127, 907-918.

Krajnović, S., Davidson, L., 2005b. Flow around a simplified car, Part 2: understanding the flow. J. Fluids Eng. 127, 919-928.

Krajnović, S., Bengtsson, A., Basara, B., 2011. Large Eddy Simulation investigation of the hysteresis effects in the flow around an oscillating ground vehicle. J. Fluids Eng. 133 (12), 121103.

Krajnović, S., Per, R., Koji, N., Branislav, B., 2012. Large Eddy Simulation of the flow around a simplified train moving through a crosswind flow. J. Wind Eng. Ind. Aerodyn. 110, 86-99.

Krajnović, S., Davidson, L., 2004. Large-Eddy simulation of the flow around simplified car model. SAE Technical Paper 2004-1-0227.

Le Good, G.M., Garry, K.P., 2004. On the use of reference models in automotive aerodynamics. SAE Technical Paper 2004-01-1308.

Lienhart, H., Becker, S., 2003. Flow and turbulence structure in the wake of a simplified car model. SAE Technical Paper 2003-1-0656.

Maskell, E.C., 1963. Theory of the blockage effect on bluff bodies and stalled wings in closed wind tunnel. RAE Report and Memoranda, 3400.

Minguez, M., Pasquetti, R., Serre, E., 2008. High-order large-eddy simulation of flow over the "Ahmed body" car model. Phys. Fluids 20, 095101. http://dx.doi.org $10.1063 / 1.2952595$

Nara, K., Tsubokura, M., Ikeda, J., Fasel, U., et. al., 2014. Numerical analysis of unsteady aerodynamics of formula car during dynamic cornering motion. In: Proceedings of the 32nd AIAA Applied Aerodynamics Conference, Atlanta, GA, USA.

Okada, Y., Nouzawa, T., Okamoto, S., Fujita, T., et. al., 2012. Unsteady vehicle aerodynamics during a dynamic steering action: 1 st report, on-road analysis. SAE Paper 2012-01-0446. doi: 10.4271/2012-01-0446.

Piomelli, U., Chasnov, J.R., 1996. Large-Eddy simulations: theory and applications, Turbulence and Transition Modelling. Springer, Netherlands, pp. 269-336.

Pope, S.B., 2000. Turbulent Flows. Cambridge University Press.

Serre, E., Minguez, M., Pasquetti, R., Deng, G.B., et al., 2013. On simulating the turbulent flow around the Ahmed body: a French-German collaborative evaluation of LES and DES. Comput. Fluids 78, 10-23.

Smagorinsky, J., 1963. General circulation experiments with the primitive equations. Mon. Weather Rev. 91 (3), 99-165.

Strachan, R.K., Knowles, K., Lawson, N.J., 2007. The vortex structure behind an Ahmed reference model in the presence of a moving ground plane. Exp. Fluids 42, 659-669. http://dx.doi.org/10.1007/s00348-007-0270-x.

Thacker, A., Aubrun, S., Leroy, A., Devinant, P., 2012. Effects of suppressing the 3D separation on the rear slant on the flow structures around an Ahmed body. J. Wind Eng. Ind. Aerodyn. 107-108, 237-243.

Toet, W., 2013. Aerodynamics and aerodynamic research in formula 1. Aeronaut. 117 (1187), 1-26.

Tsubokura, M., Ikawa, Y., Nakashima, T., Okada, Y., et al., 2012. Unsteady Vehicle Aerodynamics during a dynamic steering action: 2nd report, numerical analysis SAE. Int. J. Passeng. Cars Mech. Syst. 5 (1), doi: 104271/2012-01-0448.

Wang, X.W., Zhou, Y., Pin, Y.F., Chan, T.L., 2013. Turbulent near wake of an Ahmed vehicle model. Exp. Fluids 54, 1490. http://dx.doi.org/10.1007/ s00348-013-1490-X. 